\title{
Spinal Fluid Clotting Activity: a New Method of Evaluating Neonatal Brain Damage
}

\author{
BERNARD DALENS, ${ }^{(16)}$ MARIE-JOSĖPHE BEZOU, MAURICE COULET, JEAN-PIERRE HABERER, \\ AND GUY VANNEUVILLE \\ Department of Anesthesiology [B. D., J.-P. H., G. V.] and Department of Hematology [M.-J. B, M.C.], Hotel-Dieu, \\ Clermont-Ferrand, France
}

\section{Introduction}

The relationships between cerebrospinal fluid disturbances and neonatal cerebral injury have been emphasized by several studies $(1,6,8,9,10,11,12,13)$, including ours $(2,3,4,5)$. In particular, we have previously reported close correlations of enzyme levels in blood and CSF with neonatal brain damage as evaluated by neurologic handicaps at 1 year of age (4); threshold values were suggested to delineate high-risk from low-risk infants.

A preliminary report by Graeber and Stuart (7) described a procoagulant activity of CSF in relation to cerebral injury. This study inspired us to more thoroughly examine the CSF clotting activity of neonates according to our methodology of evaluating brain damage (4).

After several attempted assays, two kinds of tests were retained: (1) activated partial thromboplastin time, which explored the early stages of coagulation, and (2) thrombelastography, which realized a complete analysis of coagulation, allowing for subsequent reexaminations as well.

\section{MATERIALS AND METHODS}

This study was realized with neonates admitted to our neonatology center and intensive care unit. Eighty-six infants, whose major final diagnosis is shown on Table 1, were examined. Apgar scores, daily physical examinations and electroencephalographic patterns were systematically recorded.

Apgar scores at 1 min were lower than 3 in 24 cases, greater than 6 in 42 cases and intermediate in 20 cases. Neurologic examinations were quite normal in 28 cases and revealed deep abnormalities in 21 cases (prolonged coma, complete lack of primitive reflexes, seizures); they were variously (but no longer than the seventh day of life) disturbed in the 37 remaining cases. Electroencephalograms revealed paroxysmal patterns in 15 cases, normal tracings in 41 cases and nonparoxysmal abnormalities in 30 cases.

Lumbar punctures for diagnostic evaluation of cerebrospinal fluid (CSF) were done from birth to the 25 th day of life. CSF, $0.8-1.2 \mathrm{ml}$, was collected in plastic tubes and assayed within 20 min (in every case). Protein, cell counts, cultures, enzyme determinations [lactate dehydrogenase E.C. 1.1.2.7.; hydroxybutyrate dehydrogenase; creatine kinase EC. 2.7.3.2.; glutamic oxaloacetic transaminase E.C. 2.6.1.1.] were performed using standard techniques.

For the evaluation of CSF clotting activity, two mixtures were realized: (1) $0.3 \mathrm{ml}$ amount of normal pooled and platelet poor plasma (NPPP) were mixed to a blank $(0.3 \mathrm{ml}$ amount of Michaelis buffer): control mixture, and (2) $0.3 \mathrm{ml}$ amount of NPPP mixed to the same volume of CSF: assay mixture.

Measurement of activated partial thromboplastin time (APTT). Control as well as assay mixtures in $0.1 \mathrm{ml}$ amounts, were incubated at $37^{\circ} \mathrm{C}$ for $3 \mathrm{~min}$, and then respectively mixed to $0.1 \mathrm{ml}$ of cephalin $\left(1 / 10^{\circ}\right.$ dilution) and $0.1 \mathrm{ml}$ of a suspension of kaolin
(C.K. test Stago). Then $0.1 \mathrm{ml}$ amount of calcium chloride (CaCl2:0.025 mole/liter) was added and coagulation time measured at $37^{\circ} \mathrm{C}$.

Thrombelastography. Control and assay mixtures, in $0.3 \mathrm{ml}$ amounts, were distributed into different cups and placed for $3 \mathrm{~min}$ in a water bath. Next $0.06 \mathrm{ml}$ of calcium chloride $\left(\mathrm{CaCl}_{2}: 0.025\right.$ mole/liter) was added, respectively, in the two cups and recording procedure of thrombelastography performed for $60 \mathrm{~min}$. Two measurements (14) were made from the tracings (Fig. 1): (1) the reaction time $r$, which elapsed between recalcification of the plasma and the point at which the straight line divided into two lines. This line was measured $(\mathrm{mm})$ from the start to this point (film speed: $2 \mathrm{~mm} / \mathrm{min}$ ) and (2) the maximum amplitude (ma), which was the widest point of the two lines $(\mathrm{mm})$.

Three lots of samples were delineated in relation to the time elapsed since birth: (1) samples collected from birth to day 3 (DID3), (2) those collected between day 4 and day 7 (D4-D7), and (3) those collected later than day 7 (D8-D25).

All assays were performed in duplicate (and a difference no greater than $3 \%$ had been observed). The results (means and S.D.) were expressed in \% of assays versus control parameters (APTT, $\mathbf{r}$ and $\mathrm{ma}$ ) and were analyzed with Student's $t$ test. Differences were considered significant when $P<0.01$.

\section{RESULTS}

Clinical assessment of infants. The eighty-six neonates were divided into three classes in relation to brain damage according to clinical (Apgar scores, physical examinations and EEG tracings) and biologic [enzyme determinations following our previously reported (4) methodologyl patterns: (1) low-risk class (2I cases): infants with normal clinical patterns and low enzyme levels, (2) high-risk class (23 cases): infants demonstrating severe clinical abnormalities (at least two of the three clinical patterns severely disturbed) with high enzyme levels, and (3) mild-risk class (42 cases): infants demonstrating either a discrepancy between clinical and biochemical patterns or revealing mild abnormalities of clinical as well as biologic evaluations.

CSF clotting activity (Table 2). The subclasses delineated by Apgar scores $(<3,>6$, intermediate), physical examinations (normal, deeply abnormal, slightly disturbed) and EEG tracings (normal, paroxysmal, variously disturbed) did not reveal significant differences of clotting parameters.

Conversely, the three classes of infants delineated in relation to brain damage (low, high and mild-risk classes) demonstrated significant differences.

APTT (Fig. 2). All mean values were below $85 \%$, i.e., all CSF samples demonstrated a positive clotting activity versus a blank, even later than day 8. Main features of the measurements were: (1) quite constant values in every sample of low-risk infants, (2) significantly lower values in (D1-D3) samples of high-risk infants in comparison to low-risk ones, and (3) lack of significant differences in (D8-D25) samples of the three classes of infants. 
The low number of (D3-D7) samples of low-risk infants did not authorize to conclude that the smaller APTT values of highrisk infants were significantly different.

Reaction time $r$ (Fig. 3). All mean $\mathrm{r}$ values were below $75 \%$, i.e., they all revealed a procoagulant activity of CSF samples. Lowrisk and mild-risk classes did not show significant differences at any date of collection. High-risk infants demonstrated significantly smaller $r$ values in (D1-D3) samples, and significantly greater ones (i.e., less procoagulant activity) in (D4-D7) as well as (D8D25) samples; in this group, the differences observed according to the time elapsed since birth were significant.

Maximum amplitude ma (Fig. 4). The three classes of infants demonstrated significant differences in the three lots of samples. In high-risk infants, the low (Dl-D3) ma values (negative clotting activity) were followed by significantly greater ones $(>100 \%$, i.e.,

Table 1. Major final diagnosis of the neonates

\begin{tabular}{lc}
\hline \multicolumn{1}{c}{ Diagnosis } & $\begin{array}{c}\text { Number of } \\
\text { cases }\end{array}$ \\
\hline Respiratory distress & 17 \\
Convulsive and related disorders & 13 \\
Postmature infants ( $>43$ wk) & 10 \\
Toxemia with very low birth weight & 8 \\
Severe hypoglycemia & 6 \\
ABO incompatibility & 5 \\
Infection of urinary tract (without septicemia) & 4 \\
Birth injuries & 4 \\
Diarrhea due to Rotavirus & 3 \\
Shock & 3 \\
Intestinal obstruction & 3 \\
Rh sensitization & 2 \\
Acute anemia (fetal bleeding during labor) & 2 \\
Central sleep apnea & 2 \\
Dehydration due to overclothing & 1 \\
Hemophilia B with intracranial bleeding & 1 \\
Intravascular disseminated coagulopathy (without septi- & 1 \\
$\quad$ cemia) & \\
Myelomeningocele & 1 \\
\hline
\end{tabular}

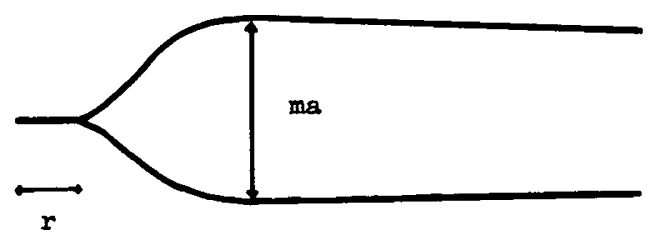

Fig. 1. Thrombelastography. Reaction time $r$ and maximum amplitude ma (film speed: $2 \mathrm{~mm} / \mathrm{mn}$ ). procoagulant activity). Low-risk infants revealed early procoagulant activity (ma $>100 \%$ ) followed by significantly lower values (ma $<100 \%$, i.e., anticoagulant activity). Concerning mild-risk neonates, the ma values were intermediate in the three lots of samples.

Influence of traumatic punctures (Table 3). Clotting parameters revealed significant but slight differences between clear $(<\mathrm{I}$ gigaerythrocyte/liter) and bloody ( $>1$ gigaerythrocyte/liter) CSF collections. APTT and $\mathrm{r}$ values were about $10 \%$ lower, and ma about $10 \%$ greater, in bloody than in clear samples (both variations representing a positive clotting activity).

\section{DISCUSSION}

The study of CSF clotting activity in three classes of neonates that were assessed according to precise evaluation of brain injury has inspired the following points.

Influence of traumatic puncture is significant and, as anticipated, blood increases clotting activity of CSF; however, the induced disturbances are quite limited, usually not exceeding 10\% of the similar activities in clear collections. Bloody collections (a frequent event in newborn populations) can be taken into account for the determination).

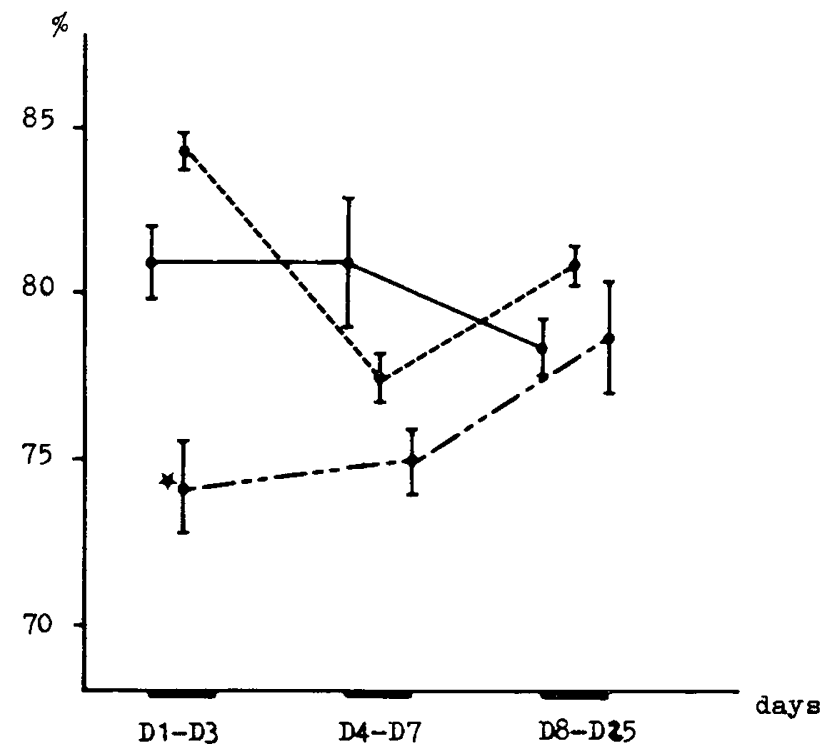

Fig. 2. APTT activity (mean and S.E.) according to the time elapsed since birth in low, mild and high-risk groups of neonates. The solid line represents the low-risk group; the broken line, the mild-risk group; and ($----)$, the high risk group.

Table 2. Clotting parameters (APTT, $r$ and $m a$ ) in the three clinical populations

\begin{tabular}{|c|c|c|c|c|c|c|c|c|c|c|}
\hline & \multicolumn{3}{|c|}{ Low-risk group } & \multicolumn{3}{|c|}{ Mild-risk group } & \multicolumn{3}{|c|}{ High-risk group } & \\
\hline & APTT & $\mathrm{r}$ & $\mathrm{ma}$ & APTT & $r$ & $\mathrm{ma}$ & APTT & $\mathrm{r}$ & $\mathrm{ma}$ & \\
\hline \multirow[t]{3}{*}{ D1-D3 } & $n^{1}$ & 10 & 10 & 10 & 16 & 16 & 16 & 9 & 9 & 9 \\
\hline & $\mathrm{m}^{2}$ & $81.13 \%$ & $49.63 \%$ & $126.00 \%$ & $84.40 \%$ & $51.93 \%$ & $89.73 \%$ & $74.28 \%$ & $45.00 \%$ & $82.43 \%$ \\
\hline & S.D. ${ }^{3}$ & $3.30 \%$ & $6.71 \%$ & $6.59 \%$ & $1.99 \%$ & $5.49 \%$ & $6.90 \%$ & $6.29 \%$ & $8.08 \%$ & $3.33 \%$ \\
\hline \multirow[t]{3}{*}{ D4-D7 } & $n$ & 3 & 3 & 3 & 10 & 10 & 10 & 6 & 6 & 6 \\
\hline & $\mathrm{m}$ & $81.00 \%$ & $56.50 \%$ & $91.50 \%$ & $77.30 \%$ & $54.00 \%$ & $119.83 \%$ & $75.00 \%$ & $63.83 \%$ & $143.30 \%$ \\
\hline & S.D. & $4.99 \%$ & $6.50 \%$ & $8.50 \%$ & $3.64 \%$ & $7.80 \%$ & $10.75 \%$ & $3.18 \%$ & $12.17 \%$ & $12.80 \%$ \\
\hline \multirow[t]{3}{*}{ D8-D25 } & $n$ & 8 & 7 & 7 & 16 & 16 & 16 & 8 & 8 & 8 \\
\hline & $\mathrm{m}$ & $78.43 \%$ & $54.57 \%$ & $90.86 \%$ & $81.00 \%$ & $58.31 \%$ & $102.13 \%$ & $78.71 \%$ & $72.13 \%$ & $115.38 \%$ \\
\hline & S.D. & $2.80 \%$ & $8.16 \%$ & $9.59 \%$ & $2.52 \%$ & $6.40 \%$ & $10.87 \%$ & $6.24 \%$ & $10.96 \%$ & $7.65 \%$ \\
\hline
\end{tabular}

\footnotetext{
${ }^{1} n=$ number of cases.

${ }^{2} \mathrm{~m}=$ arithmetic mean.

${ }^{3}$ S.D. $=$ standard deviation.
} 
On analysis of results, APTT and $r$ parameters (that evaluate the same stage of coagulation) reveal numerous similarities. In low-risk infants, the two parameters show a positive clotting activity, quite constant in regards to the time elapsed since birth (about $80 \%$ for APTT, and $50-60 \%$ for $\mathrm{r}$ parameter). Mild-risk infants do not significantly differ from low-risk ones. Concerning the high-risk group, early collections (D1-D3) reveal significantly greater positive clotting activity than in the low-risk one, as

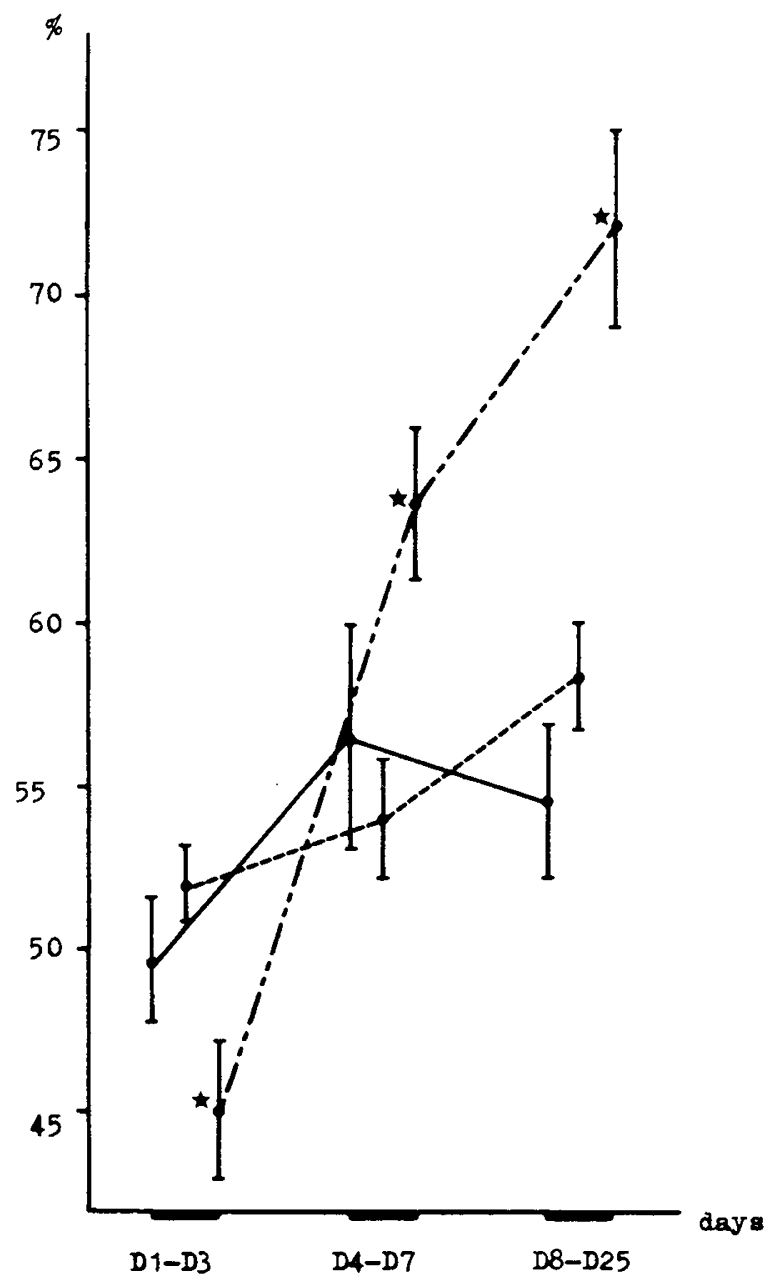

Fig. 3. Reaction time $r$ (mean and S.E.) in high, mild and low-risk groups according to the time elapsed since birth. The solid line represents the low-risk group; the broken line, the mild-risk group; and (- - _- ), the high-risk group. The asterisk represents a value significantly different from low- as well as mild-risk groups. evaluated by APTT and r parameters. Further collections (D4D7 and D8-D25) show a significant decrease in these procoagulant activities: APTT increase up to "normal" levels whereas $r$ values demonstrate a much more consequent lengthening that becomes very important past day 7 .

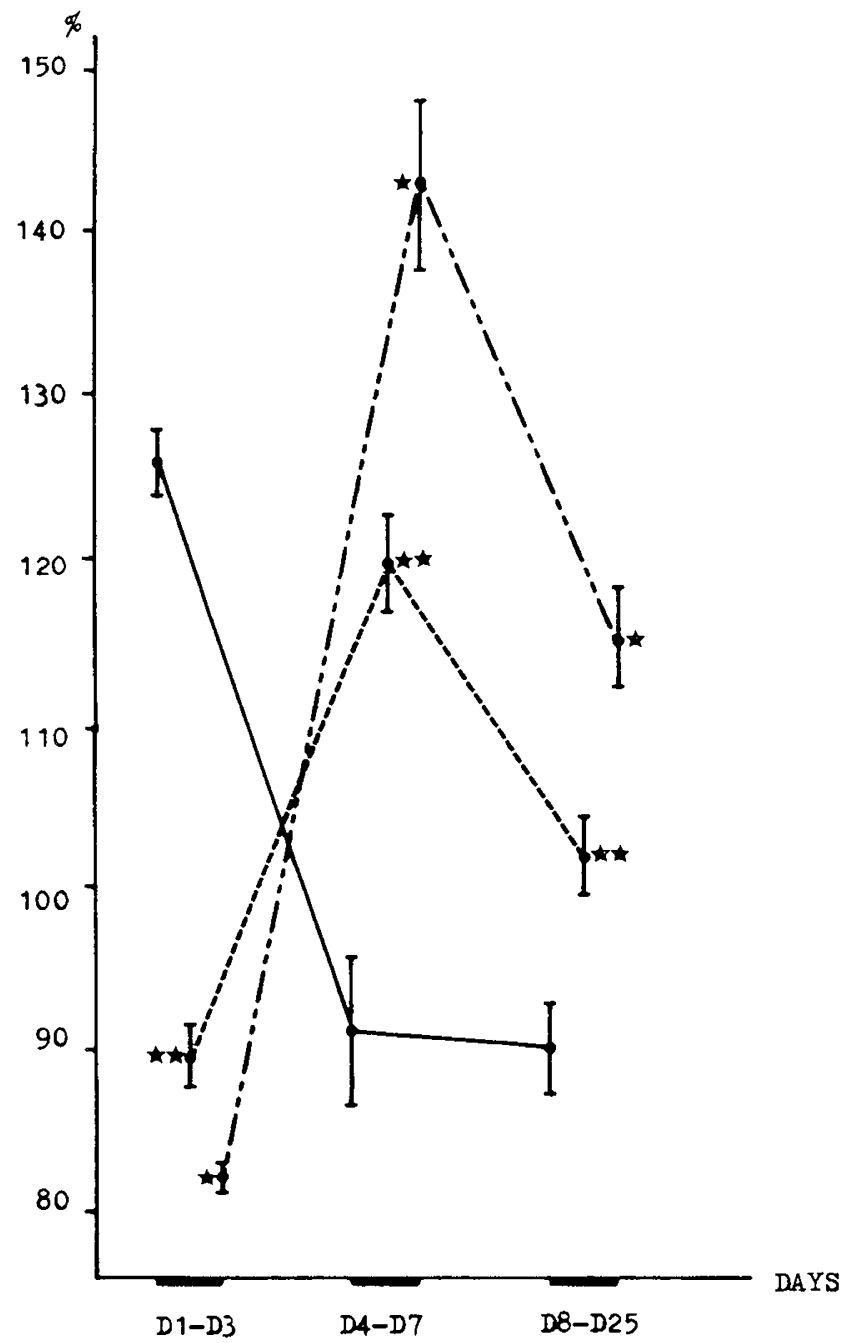

Fig. 4. Maximum amplitude ma (mean and S.E.) in high, mild and low-risk groups according to the time elapsed since birth. The solid line represents the low-risk group; the broken line, the mild-risk group; and (- - - - ), the high-risk group. Two asterisks indicate a value significantly different from the low- and high-risk groups and one asterisk, a value significantly different from the low- and mild-risk groups.

Table 3. Clotting parameters (APTT, $r$ and $m a$ ) in clear and bloody spinal fluid collections

\begin{tabular}{|c|c|c|c|c|c|c|c|}
\hline & & \multicolumn{3}{|c|}{ Clear collections } & \multicolumn{3}{|c|}{ Bloody collections } \\
\hline & & APTT & $r$ & $\mathrm{ma}$ & APTT & $r$ & $\mathrm{ma}$ \\
\hline \multirow[t]{3}{*}{ D1-D3 } & $n^{1}$ & 23 & 23 & 23 & 12 & 12 & 12 \\
\hline & $\mathrm{m}^{2}$ & $83.74 \%$ & $51.78 \%$ & $96.22 \%$ & $74.58 \%$ & $47.66 \%$ & $102.00 \%$ \\
\hline & S.D. ${ }^{2}$ & $1.67 \%$ & $4.13 \%$ & $6.79 \%$ & $3.77 \%$ & $7.98 \%$ & $8.76 \%$ \\
\hline \multirow[t]{3}{*}{ D4-D7 } & $n$ & 11 & 11 & 11 & 8 & 8 & 8 \\
\hline & $\mathrm{m}$ & $81.45 \%$ & $60.09 \%$ & $113.00 \%$ & $71.63 \%$ & $57.38 \%$ & $149.63 \%$ \\
\hline & S.D. & $3.02 \%$ & $7.99 \%$ & $11.48 \%$ & $2.18 \%$ & $9.42 \%$ & $16.92 \%$ \\
\hline \multirow[t]{3}{*}{ D8-D25 } & $n$ & 19 & 18 & 18 & 13 & 13 & 13 \\
\hline & $\mathrm{m}$ & $81.58 \%$ & $61.66 \%$ & $107.22 \%$ & $75.54 \%$ & $54.69 \%$ & $105.31 \%$ \\
\hline & S.D. & $2.67 \%$ & $5.67 \%$ & $5.96 \%$ & $3.21 \%$ & $6.72 \%$ & $10.36 \%$ \\
\hline
\end{tabular}

\footnotetext{
${ }^{1} n=$ number of cases.

${ }^{2} \mathrm{~m}=$ mean.

${ }^{3}$ S.D. = standard deviation.
} 
Maximum amplitude ma reflects the effects on fibrin synthesis (and platelet activity, but platelets were not involved here being that NPPP has been employed). Low-risk infants show increased ma values in early collections (D1-D3), rapidly decreasing in further ones (revealing a slight anticoagulant activity in comparison to a blank). Conversely, mild-risk as well as high-risk infants demonstrate significantly lower ma values in the first collection (i.e., negative clotting activity) and a considerable increase in later collections (i.e., positive clotting activity), especially between D4 and D7. One can say that high-risk versus low-risk infants show inverted variations of ma parameter according to the time elapsed since birth. Thus, APTT, $r$ and ma parameters permit a precise identification of each of the three clinical groups in regards to the time from birth.

Interpretation of these findings remains difficult. There probably exists a link between release of tissue (brain) thromboplastins and variations of CSF clotting activity.

APTT as well as $r$ parameters mainly reflect thromboplastinogenesis, and therefore, it would not be surprising that the two parameters demonstrate similar variations with time. What remains unclear is the reason for persisting positive clotting activity (could it mean that thromboplastin release persists ?) in low-risk infants, whereas high-risk ones progressively overtake what was (erroneously) anticipated to be "normal" (i.e., values obtained with a blank).

In regards to ma parameter, mild-risk infants reveal variations similar to high-risk ones (whereas they are similar to low-risk ones concerning APTT and $\mathrm{r}$ parameters). In case of low-risk infants, it can be stated that there is an early procoagulant activity in early collections with subsequent returning to normal levels (slightly lower than those obtained with a blank).

On the other hand, high-risk (and mild-risk) infants show anticoagulant activity, the meaning of which remains difficult to understand, the more so as further determinations reveal unmistakably "procoagulant" activity. It is possible that thromboplastin release is not the sole factor involved in CSF clotting activity and that local brain disorders (fibrinolysis ?) can produce interfering factors that would require further study.

\section{REFERENCES AND NOTES}

1. Cuestas, R.: Creatine kinase isoenzymes in high risk infants. Pediatr. Res., 14: 935 (1980).

2. Dalens, B., Bezou, M. J., Raynaud, E. J., Coulet, M., and Gaulme, J.: Qualitative cytology of cerebrospinal fluid as an indicator of neonatal brain damage and psychomotor outcome. Acta Paediatr. Scand., 70: 161 (1981).

3. Dalens, B., Viallard, J. L., Raynaud, E. J., and Dastugue, B.: Cerebrospinal fluid lactate and hydroxybutyrate dehydrogenase activities as indicators of neurological sequelae after neonatal brain damage. Develop. Med. Child. Neurol.. 23: 228 (1981).

4. Dalens, B., Viallard, J. L., Raynaud. E. J., and Dastugue, B.: Enzyme studies and neonatal brain damage. Acta Paediatr. Scand., 70: 743 (1981).

5. Dalens, B., Bezou, M. J., Coulet, M., and Raynaud, E. J.: Fibrin-fibrinogen degradation products in cerebrospinal fluid as an indicator of neonatal brain damage. Acta Neurol. Scand., 164: 811 (1981).

6. Drummond, M. B. and Belton, N. R.: Creatine phosphokinase (CPK) in the CSF: its value in the management of children with myelomeningocele and hydrocephalus. Arch. Dis. Child., 47: 672 (1972).

7. Graeber, J. E. and Stuart, M. J.: Spinal fluid procoagulant activity: a sensitive indicator of central nervous system damage. Lancet, 2: 285 (1978).

8. Hall, R. T.. Kulkani, P. B., Sheehan, M. B., and Rhodes, P. G.: Cerebrospinal fluid lactate dehydrogenase in infants with perinatal asphyxia. Develop. Med. Child. Neurol., 22: 300 (1980).

9. Lending, M.. Slobody, L. E., Stone. M. L., Hosbach, R. E., and Mestern, J.: Glutamic oxaloacetic transaminase and lactic dehydrogenase in cerebrospinal fluid and plasma of normal and abnormal newborn infants. Pediatrics, 24: 378 (1958).

10. Lending, M., Stone, M. L., Slobody, L. B., and Mestern, J.: Glutamic oxaloacetic transaminase and lactic dehydrogenase in pregnancy. III. Enzymatic activity in the newborn infants. Amer. J. Obs. Gynecol., 85: 41 (1963).

11. Meberg, A.. Hetland, O., Sommer, P., and Vaagenes, P.: Creatine kinase in cerebrospinal fluid in newborn infants. Clin. Chim. Acta., 85: 95 (1978).

12. Neches, W., and Platt, M.: Cerebrospinal fluid lacticodehydrogenase in 287 children including 53 cases of meningitis of bacterial and non-bacterial etiology. Pediatrics, 41: 1097 (1968).

13. Sherwin, A. L., Norris, J. W., and Bulcke, J. A.: Spinal fluid creatine kinase in neurology disease. Neurology, 19: 993 (1969).

14. Thomson, J. M.: Laboratory procedures-Thrombelastography. In: Thomson. J. M., Ed,: A practical guide to blood coagulation and haemostasis. pp. 201-204 (J. and A. Churchill, London, 1970).

15. The authors thank D. S. Royfe very much for his help in the translation of the manuscript and Dr. Raynaud for his helpful commentaries.

16. Requests for reprints should be addressed to: Dr. Bernard Dalens, Pavillon Gosselin, Hotel-Dieu BP69, 63003 Clermont-Ferrand Cedex, France.

17. Received for publication April 10, 1981.

18. Accepted for publication September 29, 1981. 